\title{
MAURÍCIO MATOS PEIXOTO (1921-2019)
}

JORGE SOTOMAYOR, RONALDO GARCIA E LUIS F. MELLO

\section{Apresentação}

Matemático de renome internacional, o Professor Maurício Matos Peixoto faleceu em 28 de abril de 2019 aos 98 anos. Ele deixou um legado incomensurável para a Matemática e, em especial, para as áreas de Sistemas Dinâmicos e Teoria Qualitativa das Equações Diferenciais.

O trabalho de Peixoto foi um elo entre a matemática clássica e o enfoque moderno, no que se refere às áreas acima citadas.

Durante sua longa carreira ele testemunhou e atuou como pioneiro em fases cruciais do desenvolvimento da matemática Brasileira. Em ordem cronólogica destacamos:

1953 - Participou da fundação do IMPA. Em torno a esse ano liderou e orientou um grupo de estudos avançados e pesquisa na Universidade do Brasil (atualmente UFRJ).

1957 - Colaborou com a organização do Primeiro Colóquio Brasileiro de Matemática. 1962 - Fundou e orientou o primeiro Seminário sobre a Teoria Qualitativa das Equações Diferenciais no IMPA. Supervisionou o primeiro grupo de pesquisa com projetos específicos que culminaram em 1964.

1971 - Organizou o primeiro Simpósio Internacional reunindo os maiores expoentes do mundo ocidental em Sistemas Dinâmicos, Salvador.

O texto contido na Seção 2 é uma tradução livre do discurso de aceitação do prêmio de Matemática da TWAS $^{1}$ outorgado a ele em 1987. Esta versão é particularmente cuidadosa na tentativa de preservar o estilo ameno e didático do autor assim como a

Data de aceitação: 22 de setembro de 2019 .

O primeiro autor contou com o apoio parcial do CNPq, Processo: PQ-SR - 307690/2016-4. O segundo autor é coordenador do Projeto PRONEX/FAPEG-CNPq Proc. 201710267000508. O terceiro autor agradece o apoio parcial da FAPEMIG, Processo: APQ-01105-18.

Os autores são gratos à Profa. A. Augusto pela doação da foto na Figura 2.

${ }^{1}$ Atualmente denominada Academia Mundial de Ciências, The World Academy of Sciences, mantendo a sigla TWAS. 

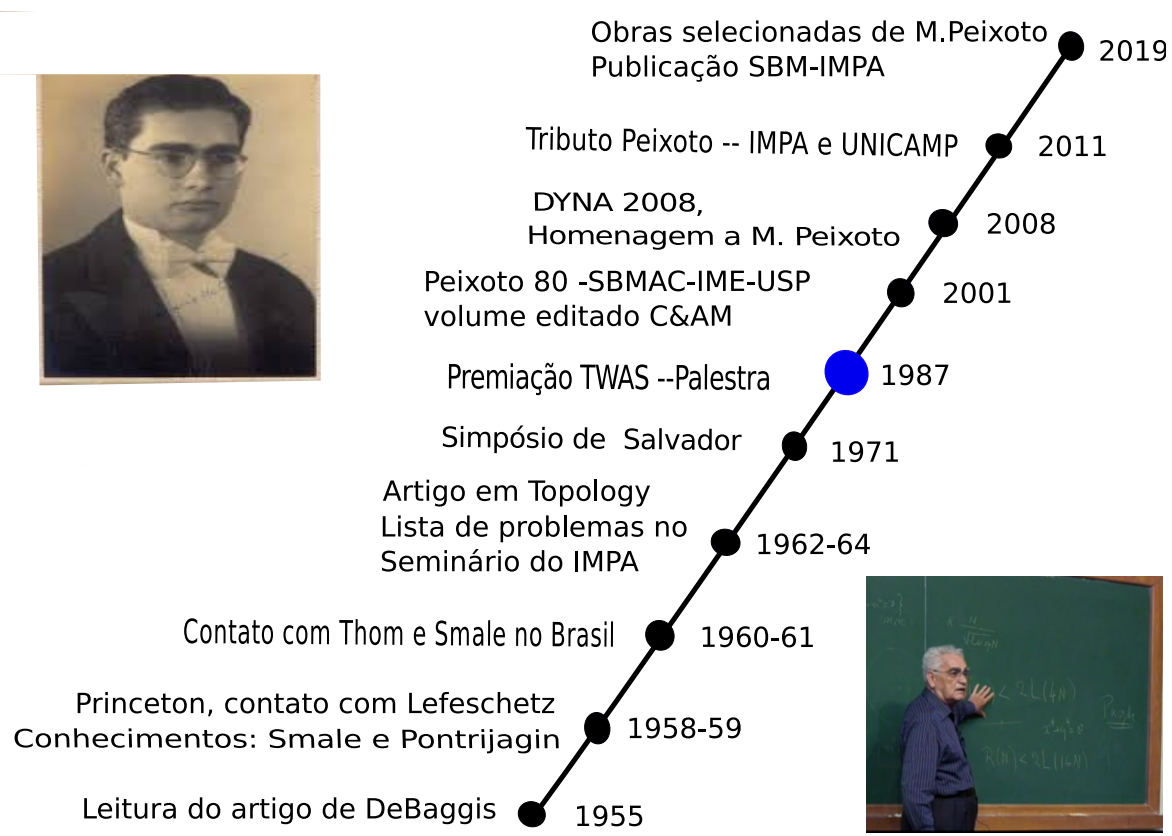

Figura 1. Alguns marcos de referência, sucintamente discutidos neste artigo, para o trabalho de M. Peixoto. Ver 3.3. Anos das fotos: superior 1936, inferior 2010.

sua exatidão matemática². Acreditamos que a sua leitura transmite uma ideia viva de parte da sua contribuição que abrange de 1955 até 1987.

Este texto constitui um marco de referência fundamental para a compreensão de uma parte crucial do frondoso domínio atual dos Sistemas Dinâmicos. Ele é um périplo fascinante que conecta a Cantor, Poincaré e Hilbert com Lefschetz, Anosov, Thom e Smale, passando pelos pioneiros russos Andronov e Pontrjagin.

Vários dos marcos de referência datados até 1987, ilustrados na Figura 1, aparecem mencionados na Seção 2. Aqueles posteriores a esse ano, assim como os não citados na Seção 2, são explicitados, sucintamente discutidos e referenciados na Subseção 3.3. Em 3.2, também incluímos comentários que julgamos ser úteis ao leitor da Seção 2 os quais aparecem anotados em notas de rodapé. Outros comentários visam dar uma ideia, mesmo que parcial, da inserção e repercussão do trabalho de M. Peixoto em Sistemas Dinâmicos e também em outras linhas de pesquisa correlatas. Veja, por exemplo, 3.4, 3.5 e 3.6.

No conjunto da obra de M. Peixoto reconhecemos a sua profundidade e ousadia intelectual, apontando em direções fecundas, correlacionadas, porém variadas. Sua trajetória esteve entrelaçada com a própria evolução da matemática brasileira, na qual atuou decisivamente.

\footnotetext{
${ }^{2} \mathrm{~A}$ presente tradução independente, direta do original, Proceedings of the Second Conference Organized by the TWAS, World Scientific, 1989, 600 - 614, corrige diversas discrepâncias tipográficas, estilísticas e matemáticas da versão publicada em Notícias, Matemática Universitária N. 8, Dezembro de 1988, $1-25$.
} 


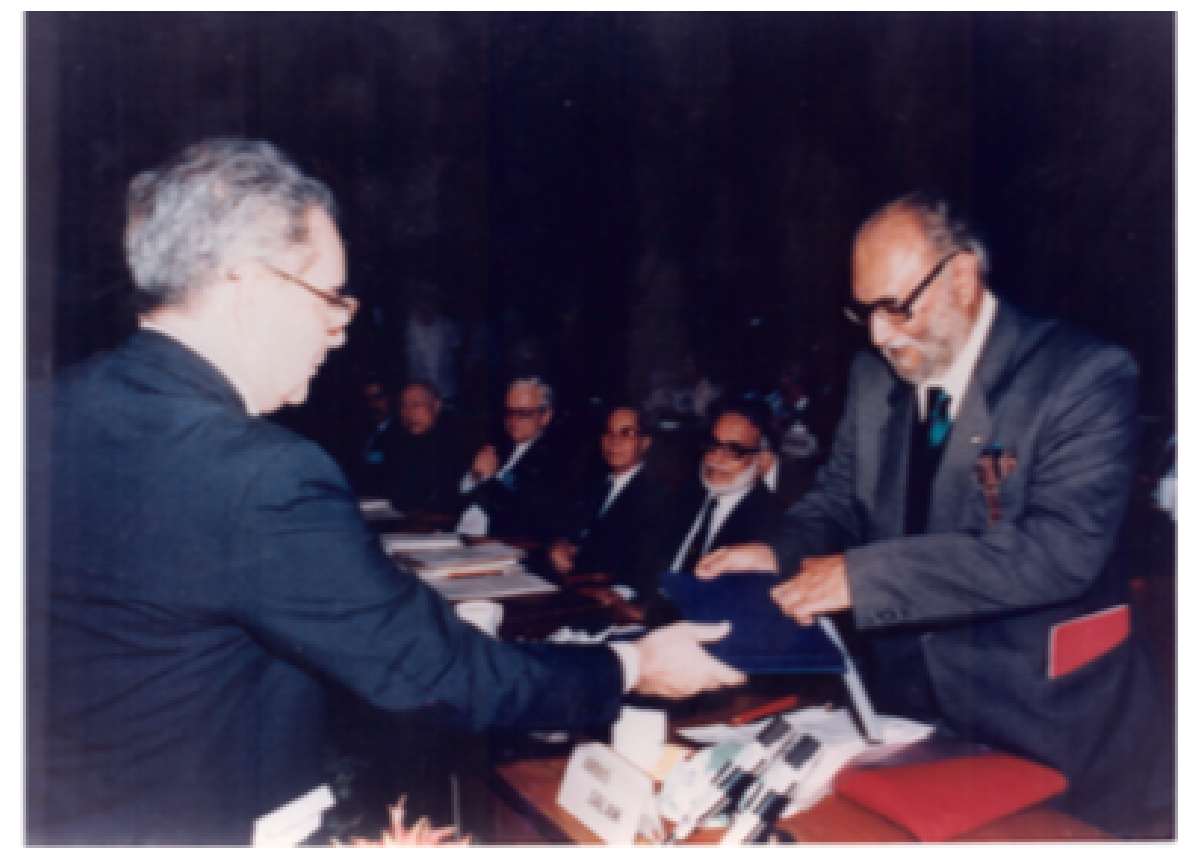

FigurA 2. M. Peixoto recebendo a medalha da premiação da TWAS de A. Salam em 1987.

\section{Palestra de M. Peixoto na Cerimônia da PremiaÇão Recebida da ACAdemia de Ciências do Terceiro Mundo}

\section{MAURÍcio PEIXOTO}

Senhoras e Senhores:

Sinto-me enormemente honrado por usar da palavra nesse encontro e é minha tarefa aqui comentar o trabalho pelo qual este Prêmio foi outorgado. Refiro-me pois, à citação lida no pódio, esta manhã pelo Prof. S. S. Chern na cerimônia de abertura e que está gravada na medalha que me foi, então, entregue pelo Presidente Abdus Salam. Nela se lê:

"Pelo seu estudo pioneiro e fundamental da estabilidade estrutural dos sistemas dinâmicos, em especial, por provar que fluxos em superfícies são genericamente estruturalmente estáveis."

Formularei aqui algumas reflexões sobre este trabalho, como se originou e de que modo contribuiu para colocar, em bases sólidas, na linguagem da Teoria dos Conjuntos, a teoria qualitativa de fluxos em variedades diferenciáveis, com objetivos razoavelmente bem definidos e problemas que apresentam uma certa unidade. $\mathrm{O}$ ponto de vista genérico aparece, então, naturalmente, e é tratado, com sucesso, em dimensão 2. Estas reflexões levaram-me a certas recordações pessoais incluídas aqui na crença de que elas possuam algum valor intrínseco ou contribuam para esclarecer o contexto do meu trabalho.

Primeiramente, gostaria de assinalar que este trabalho sobre estabilidade estrutural foi basicamente realizado em vários artigos mencionados abaixo, sendo um deles em colaboração com minha primeira esposa Marília, que não viveu para ver 
o fim dessa aventura. Foi grande, contudo, sua influência naqueles dias dourados, decisivos e já distantes.

Como mencionei anteriormente, o objeto do nosso estudo é a teoria qualitativa das equações diferenciais ordinárias, também chamadas campos vetoriais, fluxos ou sistemas dinâmicos. Esta teoria originou-se numa série de trabalhos de Poincaré [31] escritos no período 1881-1886 sob o título geral Sur les courbes définies par une équation differéntielle. Ali, pela primeira vez, considerou-se uma equação diferencial ordinária do ponto de vista da geometria do conjunto de suas trajetórias. O Teorema de Poincaré-Bendixson é típico desse ponto de vista.

Depois de Poincaré, temos um acréscimo importante à teoria qualitativa na extensa obra de G. D. Birkhoff [7], realizada nos primeiros quarenta anos do século XX. Os conjuntos $\alpha$ e $\omega$-limites e os conjuntos não errantes são alguns dos conceitos introduzidos por ele.

A estes resultados qualitativos de Poincaré e Birkhoff deve-se acrescentar a teoria da estabilidade criada por Liapounov [15] no final do século XIX. Esta teoria, notadamente analítica em seus métodos, tem também um sabor caracteristicamente qualitativo.

Em 1926, Hilbert [10, p170] escreveu a famosa declaração:

"Ninguém vai nos expulsar do paraíso que nos foi conferido por Cantor,"

rendendo assim um tributo imenso e único a um matemático.

Paraíso ou não, a frase grandiosa reflete o fato de que, desde o fim do século XIX vem ocorrendo uma tendência lenta e generalizada de situar todos os ramos da Matemática no contexto da Teoria dos Conjuntos. Em meados do século XX, essa tendência já ia avançada, com ênfase em objetos como relações de equivalência, estruturas etc.

A esse respeito evidencia-se o nome de Bourbaki, ${ }^{3}$ devido à sua corajosa tentativa de, de algum modo, controlar ou dirigir este vasto e diversificado movimento. Não obstante, a teoria qualitativa de Poincaré e Birkhoff, mantendo-se à margem desses desenvolvimentos e carente de objetivos bem definidos, perdeu seu brilho. Esclareço isso fazendo duas observações:

(I) Nenhuma estrutura - um espaço - foi construída a partir do conjunto de equações diferenciais ordinárias.

(II) Tanto Poincaré quanto Birkhoff nunca tornaram preciso o que deveria ser entendido por equivalência qualitativa de equações diferenciais.

Poincaré tinha intuições a respeito de ambos os pontos. Com relação a (I), Poincaré fala [31] em propriedades que são verdadeiras "em geral", i.e., "para a maioria dos valores" dos coeficientes, os dados sendo polinomiais. Quanto a (II), Poincaré tratou do assunto explicitamente numa conferência feita em Roma em 1908 [32]. Nela, ele frisou a necessidade de se encontrar uma "transformação", que desempenhe, em relação às equações diferenciais, o mesmo papel que as transformações birracionais têm em relação às curvas algébricas. Poder-se-ia, então, colocar na

\footnotetext{
${ }^{3}$ N.T.: https://en.wikipedia.org/wiki/Nicolas_Bourbaki, pseudônimo de um grupo de matemáticos.
} 
mesma classe todas as equações transformadas de uma dada equação. Estas são suas palavras:

"... Nós só estaremos satisfeitos quando se tiver achado um certo grupo de transformações (a exemplo das transformações de Cremona) que desempenhará em relação às equações diferenciais o mesmo papel que o grupo das transformações birracionais desempenha para as curvas algébricas. Poderemos, então, agrupar numa mesma classe todas as transformadas de uma mesma equação. Teremos ainda, por guia, a analogia com uma teoria já estabelecida, a das transformações birracionais e do gênero de uma curva algébrica."

Na linguagem de hoje, ele propunha classificar equações diferenciais módulo alguma relação de equivalência.

Tanto quanto eu saiba, esta observação de Poincaré sobre a necessidade de uma relação de equivalência para equações diferenciais não lineares gerais, uma visão penetrante, foi desconsiderada pelos autores posteriores até o dia de hoje. É provável que isto tenha ocorrido devido ao fato de que em 1908 o natural seria considerar como relação de equivalência ("a transformação") aquilo que hoje denominamos um difeomorfismo analítico. E sabemos que isso complicaria as coisas enormemente, pois haveriam demasiadas classes de equivalência.

O passo seguinte foi dado por H. Kneser [12] em um artigo datado de 1924, onde se encontra uma definição precisa da equivalência qualitativa de duas equações diferenciais. Ele considera equações diferenciais num toro $T^{2}$ e diz que duas delas, digamos $X$ e $Y$, são equivalentes se houver um homeomorfismo $h: T^{2} \rightarrow T^{2}$ que leva trajetórias de $X$ em trajetórias de $Y$. Observe-se que $h$ é apenas $C^{0}$ e opera, não nas próprias equações, mas nas suas trajetórias. Uma vez que as trajetórias são curvas orientadas, isto significa que $h$ leva curvas orientadas em curvas orientadas. Dizemos, então, que $X$ e $Y$ são topologicamente equivalentes e escrevemos $X \sim Y$.

A introdução deste conceito de equivalência topológica representa um passo decisivo em direção à colocação da teoria qualitativa no contexto da Teoria dos Conjuntos.

A equivalência topológica foi basicamente redescoberta por A. Andronov e L. Pontrjagin em 1937, numa nota fundamental de 4 páginas [2] onde eles introduzem o conceito de estabilidade estrutural.

Dada a sua importância aqui, dou a seguir, uma cuidadosa apresentação das origens desse conceito. Na nota mencionada, Andronov e Pontrjagin consideram um sistema dinâmico $x^{\prime}=P(x, y), y^{\prime}=Q(x, y)$ definido num disco $D^{2}$ no plano $(x, y)$, o campo de vetores entrando transversalmente à fronteira. Diz-se que este sistema é robusto ("grossier" porque a nota foi escrita em francês) se, dado $\varepsilon>0$, podemos encontrar $\delta>0$ tal que, cada vez que $p(x, y), q(x, y)$ e suas derivadas primeiras tenham valores absolutos menores do que $\delta$, o sistema perturbado $x^{\prime}=P+p$, $y^{\prime}=Q+q$ é topologicamente equivalente ao sistema original de tal modo que o homeomorfismo $h: D^{2} \rightarrow D^{2}$, que preserva trajetórias, seja $\varepsilon$-próximo da identidade. Supõe-se que os dados sejam analíticos. Eles, então, enunciam, sem demonstração, uma condição necessária e suficiente para que um sistema seja robusto. Usando a nomenclatura de hoje, as condições são as seguintes: 
(1) que as singularidades e as órbitas fechadas sejam hiperbólicas.

(2) que não existam trajetórias conectando pontos de sela.

As condições acima excluem a possibilidade de um comportamento complicado para as trajetórias de um sistema robusto. Em particular, existe apenas um número finito de singularidades e órbitas fechadas.

Em 1937, A. Andronov e C. Chaikin publicaram um livro em russo, Teoria das Oscilações, onde as "equações robustas" são mencionadas repetidas vezes. A obra reflete o trabalho de um grupo de pesquisadores que na União Soviética são conhecidos como a "Escola de Gorki" [4]. O livro reproduz, num apêndice, o conteúdo da nota de Andronov e Pontrjagin. Em 1949, Lefschetz dirigiu os trabalhos da tradução desse livro para o inglês e traduziu "robusto" por "estruturalmente estável".

Ao fazê-lo, Lefschetz prestou uma importante contribuição ao assunto, revelando o verdadeiro significado do novo conceito, a saber, uma interação das duas noções básicas de estabilidade e comportamento qualitativo, no sentido de equivalência topológica. Além disso, o novo termo sugere muitas outras possibilidades.

Num artigo de 1952, H. DeBaggis [6], aluno de Lefschetz ${ }^{4}$, fornece o essencial das provas que faltavam na nota de Andronov e Pontrjagin e observa que não é necessário supor que as equações sejam analíticas; $C^{1}$ é suficiente ${ }^{5}$. Foi através desse artigo que tomei conhecimento da estabilidade estrutural, num seminário da Escola Nacional de Engenharia, no Rio de Janeiro, por volta de 1955.

Em setembro de 1957, fui para a Universidade de Princeton trabalhar com Lefschetz em estabilidade estrutural. Nessa época, eu já estava convencido de que, para ir além de Andronov-Pontrjagin, era necessário adotar o ponto de vista do espaço funcional. Isto significava introduzir o espaço de Banach $\mathcal{B}$ de todos os campos vetoriais e examinar como o conjunto dos estruturalmente estáveis se situa dentro de $\mathcal{B}$. Quando expliquei isto num seminário, Lefschetz percebeu do que se tratava e se entusiasmou. Ele me interrompeu e disse:

"Agora você pode fazer todo o tipo de perguntas sobre estabilidade estrutural".

E assim foi. Comecei imediatamente a escrever as consequências desta ideia simples e, ao fim de seis meses, terminava o manuscrito do meu primeiro artigo sobre estabilidade estrutural. Lefschetz era então editor do "Annals of Mathematics" e convidou-me a publicá-lo lá. Mais tarde, ele escreveu [13, p10] que a introdução do espaço $\mathcal{B}$ e do Teorema da Densidade (6) abaixo enriqueceu enormemente o problema da estabilidade estrutural.

Naquele artigo, parti da situação no disco $D^{2}$ considerado por Andronov e Pontrjagin, e estabeleci o seguinte:

(3) Consideremos o conjunto $\mathcal{B}$ de todos os sistemas dinâmicos que atravessam a fronteira de $D^{2}$ transversalmente, o qual é aberto no espaco de Banach $\mathcal{B}^{\prime}$ de todos os campos de vetores de classe $C^{1}$ em $D^{2}$.

\footnotetext{
${ }^{4}$ N.T.: De fato, era assistente de pesquisa associado a Lefschetz. Seu doutorado foi supervisado por Karl Menger em Notre Dame, 1947.

${ }^{5}$ N.T.: A respeito deste trabalho ver o comentário em 3.2.1.
} 
(4) $\mathrm{O} \varepsilon$ que aparecia na definição de Andronov-Pontrjagin é desnecessário. Se for eliminado, ainda obtemos uma definição equivalente. Ela é simplesmente: $X \in \mathcal{B}$ é estruturalmente estável sempre que existe uma vizinhança $U$ de $X$ tal que, se $Y \in U$, então $X \sim Y$, i.e., existe um homeomorfismo de $D^{2}$ sobre si mesmo levando trajetórias de $X$ sobre trajetórias de $Y$. Esta é, hoje em dia, a definição usual de estabilidade estrutural.

(5) Estendi, então, a definição acima ao disco $n$-dimensional $D^{n}$. O conjunto $\Sigma$ de todos os sistemas estruturalmente estáveis é automaticamente aberto em $\mathcal{B}$ - este é o ponto importante da nova definição e - em seguida, provei um teorema sobre as componentes conexas de $\Sigma$.

(6) Se $n=2$, então temos que $\Sigma$, que é aberto em $\mathcal{B}$, é também denso nele.

Assim, tanto (I) como (II) mencionados acima, foram levados em consideração ou seja, temos um espaço $\mathcal{B}$ de equações diferenciais e uma relação de equivalência sobre ele, a equivalência topológica $\sim$. Os pontos interiores das classes de equivalência de $\mathcal{B}$ módulo $\sim$ são os sistemas estruturalmente estáveis. Estes, se $n=2$, têm características qualitativas muito simples e sua totalidade $\Sigma$ é aberta e densa em $\mathcal{B}$.

As consequências naturais de [22] foram [23] e [24]. Em [23], tratamos da estabilidade estrutural substituindo a condição de transversalidade na fronteira por condições mais gerais que permitem certas tangências ${ }^{6}$.

As novas condições de fronteira são densas no espaço $\mathcal{B}^{\prime}$ de todos os sistemas definidos em $D^{2}$, enquanto que a condição de transversalidade obviamente não é. Isto foi causa de uma certa perplexidade de minha parte quanto à própria definição do espaço sobre o qual se poderia provar a densidade da estabilidade estrutural.

Em [24], prova-se que em qualquer esfera $\mathbb{S}^{n}$ há infinitas classes de equivalência distintas de sistemas estruturalmente estáveis.

O trabalho descrito acima eu o realizei durante minha permanência em Princeton no período $1957-1958$.

Lefschetz ofereceu-me uma mesa no seu gabinete espaçoso e conversávamos longamente sobre Matemática, matemáticos e tudo o mais que existe debaixo do sol. Tornamo-nos verdadeiros amigos tanto quanto poderiam permitir sua eminência e, também, o fato de que, aos 73 anos, ele tinha mais do que o dobro da minha idade.

Lefschetz gostava muito de Pontrjagin e contou-me que aos 14 anos, Pontrjagin perdera as duas vistas num acidente com um fogão. O próprio Lefschetz sofrera um acidente em 1910, aos 26 anos, e tivera ambos os braços amputados pouco abaixo dos cotovelos. Decidiu, então, tornar-se matemático.

$\mathrm{Na}$ verdade, antes do verão de 1958, Lefschetz encontrara Pontrjagin apenas em algumas ocasiões durante um congresso em Moscou no começo da década de trinta. Conversaram longamente sobre Matemática e Lefschetz ficou tremendamente impressionado. Referia-se ao jovem Pontrjagin daqueles dias em termos hiperbólicos:

"Tudo o que ele tocava tornava-se ouro."

${ }^{6}$ N.T.: Veja comentários pertinentes a estes artigos em 3.2.2. 
Lefschetz, septuagenário, mesmo não sendo nenhum Midas ${ }^{7}$, era capaz de ter intuições profundas e vitais e de expressá-las de maneira encantadora. Uma vez, antes de encontrar Smale, eu disse a Lefschetz que:

"O problema com a estabilidade estrutural é que ninguém se importa com ela."

E ele disse:

"Não Maurício, isso não é problema, é sorte sua. Trate de trabalhar o mais rápido e duramente que puder nesse assunto porque o dia virá em que você não vai compreender uma só palavra do que eles estarão dizendo sobre estabilidade estrutural. Isso aconteceu comigo em Topologia."

Mas nem todos viam a coisa dessa forma. Durante o Congresso Internacional de Matemáticos de Edinburgh, em 1958, Lefschetz tomou a iniciativa de promover um encontro meu com Pontrjagin e ofereceu-se para servir de intérprete inglês-russo. A ideia era que eu comunicasse a Pontrjagin o conteúdo de [23] que não fora ainda publicado.

Do meu ponto de vista, o encontro foi pouco produtivo. Pontrjagin não estava interessado no assunto, alegando que a estabilidade estrutural não poderia existir além do disco $D^{2}$. Em particular, não poderia existir nem mesmo no toro $T^{2}$ porque no fluxo linear correspondente o tipo topológico mudaria se o coeficiente angular mudasse de racional para irracional.

Ignorava ele que sua própria criatura já abria as asas e muito em breve estaria voando alto e para longe.

Um matemático que acreditou na estabilidade estrutural foi $\mathrm{H}$. Whitney que, a seu modo reservado, deu uma contribuição substancial na redação do meu artigo "On structural stability", [23]. A prova de um dos lemas me foi comunicada por ele. Só muitos anos mais tarde é que percebi que dois anos antes disso, em 1955, para aplicações no plano, Whitney provara um teorema que, hoje, pode ser expresso afirmando-se que as aplicações estruturalmente estáveis formam um subconjunto aberto e denso do espaço de todas as aplicações diferenciáveis.

Outro matemático que acreditava na estabilidade estrutural, e que se tornaria a figura central da teoria qualitativa das equações diferenciais ordinárias, foi Steve Smale. Fui apresentado a ele por Elon Lima em Princeton no verão de 1958 logo depois que voltei de Edinburgh. Antes disso, desde algum tempo atrás, e através do próprio Lima, eu já estava ciente de que Smale era uma grande promessa. Mais tarde, em notas evocativas, Smale escreveu sobre nosso encontro em Princeton [42, p148] e descreve sua reação ao tomar conhecimento dos resultados que deveriam aparecer no meu artigo no Annals:

"Através de Lefschetz, Peixoto interessou-se por estabilidade estrutural e mostrou-me seus próprios resultados sobre a estabilidade estrutural no disco (num artigo que deveria aparecer no "Annals of Mathematics, 1959"). Entusiasmei-me imediatamente, não só pelo

${ }^{7}$ N.T.: Midas foi um rei lendário da Frígia, na Ásia Menor. 
que ele estava fazendo, mas pela possibilidade de que, usando minha experiência em Topologia, eu pudesse estender seu trabalho à dimensão $n^{\prime \prime}$.

Assim começou o maginífico trabalho de Smale sobre sistemas dinâmicos, um trabalho que será lembrado aqui, apenas de passagem e na medida em que ele ajuda a dar alguma perspectiva ao meu.

Smale foi rápido em assinalar que todos os fatos anteriores sobre o disco $D^{2}$ podiam ser expressos sobre a esfera $\mathbb{S}^{2}$ sem mais nada a adicionar. Eu já estava certo disso, mas, nunca tendo trabalhado anteriormente em mais de um sistema de coordenadas, fiquei um tanto perplexo e me engajei em provas complicadas.

Quando Smale me disse, que a coisa era direta, perguntei: "Direta, como?" e ele respondeu: "Direta como uma flecha!"

Isso descreve bem o modo como vi Smale entrar nessa área: seu talento para detectar os pontos essenciais, a força, tenacidade e a velocidade que ele evidenciou ao colocar seus conhecimentos de Topologia Diferencial na direção da Estabilidade Estrutural.

Podemos resumir os resultados mencionados acima em $\mathbb{S}^{2}$ da seguinte maneira: seja $\chi=\chi\left(\mathbb{S}^{2}\right)$ o espaço de todos os fluxos em $\mathbb{S}^{2}$ com a topologia $C^{1}$. Um tal fluxo é estruturalmente estável se, e somente se, as condições (1) e (2) acima forem satisfeitas. Além disso, se $\Sigma$ for o conjunto de todos esses fluxos, então

(7) $\Sigma$ é aberto e denso em $\chi$;

(8) As classes de equivalência de $\Sigma$, módulo , são passíveis de classificação.

Assim, obtivemos uma compreensão mais profunda das características qualitativas de todos os fluxos de $\mathbb{S}^{2}$. Na verdade, temos o espaço $\chi$ de todos os fluxos e uma relação de equivalência $\sim$ nele, bem formulada na linguagem da Teoria dos Conjuntos. O problema de descrever ou classificar as classes de equivalência de $\chi$, módulo , aparece, então, de forma natural. Entretanto, este é um problema sem esperanças. Uma vez que qualquer conjunto fechado pode ser o conjunto de singularidades de algum elemento de $\chi$, nosso problema é mais complicado do que a classificação topológica dos conjuntos fechados em $\mathbb{S}^{2}$, um problema intratável.

Com a estabilidade estrutural, entretanto, tudo se torna claro, no modo acima descrito: $\Sigma$ é, num certo sentido, "quase todo" o $\chi$ e as classes de equivalência de $\Sigma$, módulo $\sim$, são, perfeitamente tratáveis, embora as de $\chi$ módulo $\sim$, não o sejam.

Meu próximo passo foi estender este trabalho a variedades diferenciáveis compactas de dimensão 2. Seja $\chi=\chi^{r}\left(M^{2}\right), r \geq 1$, o espaço de todos os fluxos em $M^{2}$ com a topologia $C^{r}$ e seja $\Sigma$ a totalidade dos fluxos estruturalmente estáveis. Em 1962 [26], apresentei a prova do seguinte:

Teorema. Os fluxos estruturalmente estáveis são caracterizados pelas condições de Andronov-Pontrjagin acima mencionadas (1), (2) e mais a condição

(9) os conjuntos $\omega$ e $\alpha$-limite de qualquer trajetória são ou uma singularidade ou uma órbita fechada.

Além disso, $\Sigma$ é aberto e denso em $\chi$. 
Este resultado será referido daqui por diante simplesmente como o Teorema ${ }^{8}$. Essencialmente, ele constitui o objeto desta palestra. Até agora, tentei explicar seus antecedentes. No que segue, prestarei alguns esclarecimentos técnicos e explicarei como se articula com os desenvolvimentos posteriores, dando, assim, algumas perspectivas sobre seu significado.

A principal dificuldade para provar este teorema deriva do "closing lemma". Este problema, introduzido naquele mesmo artigo [26], pode ser formulado da seguinte maneira: dada uma trajetória recorrente não-trivial $\gamma$ de um fluxo $X$ e um ponto $p \in \gamma$, então é possível encontrar uma pequena perturbação $X^{\prime}$ na topologia $C^{r}$, tal que a trajetória de $X+X^{\prime}$ por $p$ seja fechada. O $C^{0}$-"closing lemma" é então verdadeiro e trivial, mesmo em dimensão $n$. Trata-se de um problema inteiramente local.

No caso $M^{2}=T^{2}$ e se $X$ não contiver singularidades, o "closing lemma" $C^{r}, r \geq 1$, é relativamente simples e foi provado em [26]. Nos outros casos nunca consegui proválo, o problema continua em aberto. O que provei em [26] foi algo mais fraco, uma alternativa: se $M^{2}$ é orientável e as singularidades de $X$ são hiperbólicas (nenhuma restrição neste contexto), então a trajetória de $X+X^{\prime}$ por $p$ será fechada ou une dois pontos de sela. Isto é suficiente para provar o Teorema. A prova é, então, válida para $M^{2}$ orientável e topologia $C^{r}, r \geq 1$. Ora, naquele mesmo artigo [26], fiz uma análise que pretende cobrir igualmente o caso em que $M^{2}$ é não-orientável, $r \geq 1$, mas esta parte do meu argumento não está correta, como Charles Pugh me chamou a atenção em 1968. Até agora este erro ainda não foi corrigido. O caso nãoorientável está coberto pelo $C^{1}$ - "closing lemma" de Pugh [33]. Assim, no momento, o Teorema, para $r=1$, está provado para qualquer $M^{2}$ orientável ou não. Para $r \geq 1$, está provado para $M^{2}$ orientável. É fácil perceber que o argumento utilizado para o caso orientável também cobre o caso em que $M^{2}$ é a garrafa de Klein [18].

Carlos Gutierrez [8] demonstrou que este é também o caso quando $M^{2}$ é o toro com um "cross-cap". No plano projetivo toda recorrência é trivial. Em todos os outros casos não orientáveis, o Teorema nunca foi provado para $r>1$.

Carlos Gutierrez [9] mostrou que, no caso $r=2$, permitindo apenas uma perturbação local e não fazendo restrições sobre a natureza das singularidades, o $C^{2}$-"closing lemma" é falso. Colocando em outras palavras, é impossível provar o $C^{2}$-"closing lemma" por meio de uma perturbação local.

Saliento que a classe de diferenciabilidade é um assunto importante. Na verdade, em se tratando de fenômenos de bifurcação, tem-se que considerar $r \geq 4$, [43, 28].

Cabe aqui fazer uma observação de caráter histórico sobre a caracterização acima e o Teorema da densidade. Muitos anos após sua publicação em 1962, tomei conhecimento, através de traduções feitas do russo, de que para o caso mais simples e particular em que $M^{2}=T^{2}$ e os fluxos não tendo singularidades, a caracterização da estabilidade foi dada por Pliss em 1960 [30] e a densidade por Arnold, em 1961 [5]. Neste último caso, o "espaço" é plenamente reconhecido ${ }^{9}$.

\footnotetext{
${ }^{8}$ N.T.: Para uma apresentação didática deste teorema veja [8, cap. 4].

${ }^{9}$ N.T.: Entendemos que o autor quis dizer que na literatura russa sobre Estabilidade Estrutural, esta é a primeira que faz menção de um espaço funcional de Sistemas Dinâmicos.
} 
Voltando ao Teorema, observa-se que as condições (1), (2) e (9) implicam que numa $M^{2}$ compacta, fluxos estruturalmente estáveis são similares a aqueles que o são em $\mathbb{S}^{2}$ e, assim, as classes de equivalência correspondentes são passíveis de classificação. Na verdade, só muito mais tarde, em 1973, [25], é que fiz uma classificação completa, baseada no meu trabalho de 1959 [23].

Tomando emprestada uma nomenclatura há muito usada na Geometria Algébrica, é hoje comum dizer que uma propriedade de um fluxo é genérica se for satisfeita por todos os fluxos de um conjunto de Baire (em particular, aberto e denso) de fluxos de $\chi$. A razão para esta definição é que aberto e denso é uma propriedade forte demais em dimensões superiores a 2. A parte da densidade do Teorema diz que fluxos em $M^{2}$ são genericamente estruturalmente estáveis, como referido na citação gravada na medalha da premiação. Assim, o Teorema oferece uma visão boa do espaço $\chi=\chi\left(M^{2}\right)$ dos fluxos em $M^{2}$, uma vez que o conjunto $\Sigma$ dos que são estruturalmente estáveis é bastante grande dentro de $\chi$ e que, para cada elemento de $\Sigma$, temos um bom conhecimento do comportamento do fluxo em toda a superfície $M^{2}$. Trata-se, realmente, de um teorema de análise global. E temos, enquadrada dentro da Teoria dos Conjuntos, uma linguagem apropriada para formular problemas gerais.

Por exemplo, o estudo de bifurcações a 1-parâmetro (de fluxos em $M^{2}$ ) pode ser considerado como o estudo da interseção de um arco em $\chi \operatorname{com} \Sigma$. Jorge Sotomayor trabalhou nesse assunto na sua tese no IMPA [43]. Mais uma vez, temos que adotar o ponto de vista genérico, perturbando o arco. Da mesma forma, pode-se fazer o estudo das bifurcações a 2-parâmetros e assim por diante ${ }^{10}$.

Direi agora algumas palavras para explicar como o trabalho acima, em $M^{2}$, influenciou o desenvolvimento subsequente da teoria qualitativa de fluxos numa variedade diferenciável compacta $M^{n}, n>2$. Tal como antes, temos o espaço dos fluxos, $\chi=\chi^{r}\left(M^{n}\right), r \geq 1$, com a topologia $C^{r}$ e o conjunto $\Sigma$ de todos aqueles estruturalmente estáveis. Aqui, encontramos uma situação bem mais complexa do que a presente em duas dimensões, com fenômenos inteiramente novos entrando em cena. Como consequência, para $n>2$, não ocorre nada de tão preciso e simples como no Teorema.

Daí resulta um problema natural que é o de expressar as condições (1), (2) e (9) de modo que sejam válidas para dimensão $n>2$ e, ao mesmo tempo, as propriedades correspondentes sejam genéricas, i.e., os fluxos que as satisfaçam formem um conjunto de Baire em $\chi$.

A condição (1) permanece inalterada no caso $n>2$. Quanto à condição (2), a maneira correta de expressá-la é:

(2') As variedades estáveis e instáveis das singularidades e órbitas fechadas se intersectam transversalmente. Este fato foi descoberto por Smale [37], no começo de 1959, e apontou para a estreita relação entre o nosso assunto e a teoria da transversalidade que Thom introduziu na Topologia Diferencial.

O artigo de Thom de 1949 [44] está relacionado com isto.

O fato que as propriedades (1) e (2') são genéricas constitui o Teorema de KupkaSmale [27]. Foi esse o tema da tese de Ivan Kupka, no IMPA, em 1964.

\footnotetext{
${ }^{10}$ N.T.: Ver a este respeito os comentários em 3.2.3.
} 
Observe-se que, para $n>2$, um fluxo pode satisfazer (1), (2') e exibir uma infinidade de órbitas fechadas.

Quando existir apenas um número finito de órbitas fechadas, temos os fluxos introduzidos por Smale no artigo acima [37] e denominados fluxos de Morse-Smale por Thom, no começo da década de 1960. Os fluxos de Morse-Smale existem em qualquer $M^{n}, n \geq 2$, e foi provado, em 1970, num importante artigo de Jacob Palis e Smale [21], que eles são estruturalmente estáveis. Assim, em qualquer variedade, $\Sigma$ é não vazio.

Agora que se têm as condições (1) e (2') na dimensão $n \geq 2$, pode-se desejar estender, da mesma forma, a condição (9). Seja $\Gamma$ a união de todas as singularidades e órbitas fechadas de um fluxo e $\Omega$ seu conjunto não errante. Lembremo-nos de que um ponto em $M^{n}$ pertence a $\Omega$ quando qualquer vizinhança dele, transformada pelo fluxo, intersecta sua vizinhança inicial para valores do tempo arbitrariamente grandes. Assim, o que toma o lugar de (9) é

(9') $\Omega=\bar{\Gamma}$.

Que esta propriedade seja genérica na topologia $C^{1}$, constituti um belo e difícil teorema de Pugh [34], consequência do seu "closing lemma".

Se $n=2,(1)$, (2) e (9') são equivalentes a (1), (2) e (9); nesse sentido, (9') é uma generalização de (9). O fato de que as propriedades (1), (2') e (9'), tomadas em conjunto, sejam uma propriedade genérica $C^{1}$ é normalmente mencionado como o Teorema da Densidade Geral. Agora que foram estendidas a $n>2$ as propriedades que para, $n=2$, são características da estabilidade estrutural, é natural formular para $n>2$ os seguintes problemas:

Problema (i): caraterizar os fluxos estruturalmente estáveis em $M^{n}$.

Problema (ii): $\Sigma$ será denso em $\chi$ ?

O Problema (ii), na topologia $C^{r}, r \geq 1$, tem uma resposta negativa dada por Smale em 1966, [39]: há uma certa $M^{4}$ na qual a estabilidade estrutural não é uma propriedade genérica. Em 1970, R. Williams [45] provou que o mesmo ocorre numa certa $M^{3}$. Assim, o caráter genérico da estabilidade estrutural de fluxos é um fenômeno de 2 dimensões. Caso $M^{n}$ seja não compacta, para fluxos, mesmo quando $n=2$, a estabilidade estrutural deixa de ser uma propriedade genérica [29]. O argumento é semelhante aos usados nos exemplos acima. Considerarei sempre $M^{n}$ compacta.

O Problema (i), para difeomorfismos e com a topologia $C^{1}$, foi resolvido recentemente, após os esforços de vários matemáticos, tal como indicado abaixo.

Aqui, será necessário fazer uma digressão a respeito dos difeomorfismos. Primeiramente, deve-se observar que o que foi feito acima para os fluxos pode ser facilmente traduzido para a linguagem dos difeomorfismos de uma variedade compacta diferenciável $M^{n}$.

Seja $\operatorname{Diff}^{k}\left(M^{n}\right)$ o espaço dos difeomorfismos de $M^{n}$ com a topologia $C^{k}$. Um tal difeomorfismo $f$ é dito estruturalmente estável se existir uma vizinhança $U$ de $f$ tal que, sempre que $g \in U$ se tenha $h g=f h, h$ homeomorfismo de $M^{n}$ em $M^{n}$. Os difeomorfismos $f$ e $g$ são ditos conjugados.

O estudo de um difeomorfismo em $M^{n}$ é o mesmo que o estudo de um certo fluxo numa certa $M^{n+1}$, o fluxo suspendido. Em muitos casos, o que pode ser feito 
para os difeomorfismos em $M^{n}$, pode ser transposto para fluxos em $M^{n+1}$, sem muitas dificuldades adicionais. Há boas razões para se estudar meticulosamente os difeomorfismos como tais.

O Teorema acima aplica-se apenas quando $M^{n}=\mathbb{S}^{1}$ e afirma que os difeomorfismos estruturalmente estáveis de $\mathbb{S}^{1}, \quad \Sigma$, são caracterizados por terem apenas um número finito de pontos periódicos, todos hiperbólicos, e que $\Sigma$ é denso em $\operatorname{Diff}^{k}\left(\mathbb{S}^{1}\right)$, $k \geq 1$.

Um divisor de águas na teoria qualitativa foi a descoberta de Smale de que seu difeomorfismo tipo ferradura em $\mathbb{S}^{2}$, contendo infinitos pontos periódicos e homoclínicos, é estruturalmente estável [38]. O comportamento homoclínico foi descoberto por Poincaré, na década de 1890, no problema dos 3 corpos. Ele apontou as características qualitativas altamente complicadas na dinâmica do citado difeomorfismo. Nas mesmas linhas, encontra-se o notável trabalho de D. Anosov [3] provando, entre outras coisas, que o fluxo geodésico em superfícies de curvatura negativa, considerado por Hadamard nos anos 1890, é estruturalmente estável. Assim, Smale e Anosov ampliaram consideravelmente o escopo do conceito de estabilidade estrutural, mostrando que ele poderia assimilar algumas dessas formas complexas de recorrência que eram mal compreendidas.

Com relação à coexistência da estabilidade estrutural com a infinidade dos pontos periódicos, cabe uma nota histórica. Num artigo inicial, Smale [37] havia formulado sua expectativa de que os fluxos Morse-Smale fossem densos em $\chi(M)$. Levinson, numa carta a Smale, observou que o trabalho de Cartwright-Littlewood, além do dele próprio sobre a equação de Van der Pol com uma perturbação periódica, oferece um contra exemplo que mostra, num espaço a três dimensões, um fluxo com uma infinidade de órbitas periódicas que não podem desaparecer por uma pequena perturbação $C^{1}$. A ferradura de Smale é uma conceitualização desta situação. Imediatamente após o difeomorfismo da ferradura, Thom divulgou verbalmente o seu exemplo de difeomorfismo $f$ no toro, explicado a seguir. A aplicação linear $\bar{f}$ em $\mathbb{R}^{2}$, definida pela matriz unimodular $\left(\begin{array}{ll}2 & 1 \\ 1 & 1\end{array}\right)$, induz o difeomorfismo $f: T^{2} \rightarrow T^{2}$. A origem é um ponto fixo de $f$ e as variedades estáveis e instáveis correspondentes têm inclinações irracionais, são densas em $T^{2}$ e encontram-se transversalmente. Assim, os pontos homoclínicos e periódicos de $f$ são densos em $T^{2}$. Este é, talvez, o exemplo mais facilmente compreendido de um difeomorfismo estruturalmente estável com infinitos pontos periódicos. O exemplo de Thom é o mais simples daquilo que hoje é denominado um difeomorfismo de Anosov. Ele teve importante papel no trabalho de Smale e Anosov, ver [40, p764], [42, p151], [4, p86].

Voltando ao Problema (i), para difeomorfismos, começamos com o importante trabalho de Smale - Sistemas Dinâmicos Diferenciáveis [40] - no qual se estabeleceu uma teoria geral de difeomorfismos em uma variedade diferenciável compacta $M-$ incorporando as ideias de genericidade, comportamento qualitativo e estabilidade que provaram ser tão bem sucedidas em pequena dimensão. O objetivo original estabelecido neste artigo [40, p747] era obter algum tipo de análogo do Teorema acima, para $n>1$, com respeito a alguma relação de equivalência mais fraca do que 
a conjugação, mas preservando, de algum modo, a estrutura de órbitas. Este objetivo foi abandonado há muito tempo já que nunca foi encontrada uma propriedade genérica que preserve num sentido razoável a estrutura das órbitas. Ver [41] e [40, nota 2]. Ainda assim, esse artigo [40] permanece no cerne da teoria qualitativa das equações diferenciais ordinárias e sua influência é tão penetrante que, depois dele, a área passou a denominar-se "Sistemas Dinâmicos".

Alguns dos conceitos mais profundos da teoria qualitativa, tais como o Axioma $A \mathrm{e}$ os conjuntos básicos, devem-se a Smale. A ele devemos também a $\Omega$-estabilidade e a condição de transversalidade forte que é uma condição mais natural do que o axioma $B$, introduzido originalmente em [40]. Na teoria de Smale, o objeto de importância primordial é o conjunto não errante $\Omega=\Omega(f)$, associado a cada $f \in \operatorname{Diff}(M)$. Por definição, $p \in \Omega$ quando, dada qualquer vizinhança $U$ de $p$, há uma infinidade de inteiros $m$ com $U \cap f^{m}(U) \neq \emptyset$. Naturalmente $\Omega$ é fechado e invariante: $f(\Omega)=\Omega$.

Agora damos duas definições cruciais. Um difeomorfismo $f$ determina uma estrutura hiperbólica em $\Omega=\Omega(f)$ (ou em qualquer conjunto invariante) quando houver uma decomposição invariante contínua do fibrado tangente $T M$, restrito a $\Omega, T M \mid \Omega=E^{s} \oplus E^{u}$ de tal modo que $D f$ seja uma contração em $E^{s}$ e uma expansão em $E^{u}$, i.e., existem constantes $c>0,0<\lambda<1$, tais que $\left|D f^{m}\right| E^{s}(x) \mid \leq c \lambda^{m}$, $\left|D f^{-m}\right| E^{u}(x) \mid \leq c \lambda^{m}$, para todo $x \in \Omega$ e $m \geq 0$. As normas são tomadas em alguma métrica Riemanniana.

Diz-se que um difeomorfismo $f$ satisfaz o axioma $A$ quando determina uma estrutura hiperbólica em $\Omega$ e, além disso, os pontos periódicos de $f$ são densos em $\Omega$ (como no teorema de Pugh). Um difeomorfismo de Morse-Smale obviamente verifica o axioma $A$.

Um teorema fundamental de Smale - o Teorema de Decomposição Espectral diz que, quando $f$ satisfaz o axioma $A$, há uma única decomposição finita de $\Omega=$ $\Omega_{1} \cup \ldots \cup \Omega_{m}$ em conjuntos fechados, disjuntos e invariantes $\Omega_{i}$, os conjuntos básicos, em cada um dos quais $f$ é topologicamente transitivo, i.e., há uma órbita densa.

Outra consequência importante do axioma $A$ é o fato de que para qualquer $x \in M$, podemos associar uma variedade estável $W^{s}(x)$ e uma variedade instável $W^{u}(x)$, definidas por

$$
\begin{gathered}
W^{s}(x)=\left\{y \in M: d\left(f^{m}(x), f^{m}(y)\right) \rightarrow 0 \text { quando } m \rightarrow \infty\right\}, \\
W^{u}(x)=\left\{y \in M: d\left(f^{-m}(x), f^{-m}(y)\right) \rightarrow 0 \text { quando } m \rightarrow \infty\right\} .
\end{gathered}
$$

Estas são subvariedades injetivamente imersas [11, 40]. Se para todo $x \in M, W^{s}(x)$ é transversal a $W^{u}(x)$ no ponto $x$, diz-se, então, que $f$ satisfaz a condição de transversalidade forte. Ora, a solução do Problema (i) começou com a conjectura de Palis-Smale [21] de que um difeomorfismo f é estruturalmente estável se, e somente se, satisfaz o axioma $A$ e a condição de transversalidade forte.

Com o trabalho de J. Robbin [35] e C. Robinson [36], no começo da década de 70, o problema ficou reduzido a provar que a estabilidade estrutural implica o axioma $A$, a chamada conjectura da estabilidade. Para uma $M$ arbitrária, $r=1$, esta conjectura foi provada por S. D. Liao [14] em 1980 e por Ricardo Mañé em 1982 [16]. Mas, o argumento de Mañé já continha o germe da sua notável prova da conjectura da estabilidade para uma dimensão arbitrária, $r=1$ [17], que foi 
completada há alguns meses. Logo depois, Palis [20] demonstrou que o argumento de Mañé poderia ser adaptado para provar uma conjectura de Smale caracterizando os difeomorfismos $\Omega$-estáveis. Um difeomorfismo $f \in \operatorname{Diff}(M)$ é $\Omega$-estável quando existir uma vizinhança $U$ de tal $f$ tal que sempre que $g \in U$ haverá, então, um homeomorfismo $h: \Omega(f) \rightarrow \Omega(g)$ com $g h=h f$.

Uma observação final sobre o Problema (ii): desde que Smale provou que a estabilidade estrutural não é uma propriedade genérica, é natural perguntar sobre a genericidade da $\Omega$-estabilidade e do axioma $A$. Abraham e Smale [1] provaram que a $\Omega$-estabilidade não é genérica e S. Newhouse [19] provou que o axioma $A$ também não é uma propriedade genérica.

Assim o espaço $\operatorname{Diff}\left(M^{n}\right), n>1$, é grande demais e parece não haver nele um subconjunto de Baire que tenha características qualitativas simples de se descrever.

Com isto, termino a apresentação de alguns desenvolvimentos relativos ao Teorema.

A descrição dada acima sobre as origens deste Teorema e os desenvolvimentos a ele relacionados estaria incompleta e injusta sem uma referência especial a René Thom. Conheci-o através de Smale, quando este passava o primeiro semestre de 1960 no IMPA. Naquela época, eu começava a escrever o Teorema [26]. Em junho, Smale fez uma viagem rápida a Zurich para participar de uma conferência e falar do seu trabalho, sobre a conjectura de Poincaré em dimensão superior, que ele estava terminando. Quando voltou, eu lhe disse que estava em dificuldades com o que hoje se chama "closing lemma". Ele respondeu que, na conferência, encontrara Thom que tivera a mesma dificuldade em dimensão $n$ quando lidava com outro problema. Esta foi a origem de extensa correspondência que iniciei com Thom sobre este e outros assuntos. A finalização do Teorema em [26] - basicamente um corpo a corpo com o "closing lemma" - que muito devo a Thom, foi completada enquanto ele passava um mês no IMPA em 1961. Ele era editor da recém fundada revista "Topology" e sugeriu-me que o publicasse lá, [26].

\section{REFERÊNCIAS}

[1] R. Abraham and S. Smale. Nongenericity of $\Omega$-stability. In Global Analysis (Proc. Sympos. Pure Math., Vol. XIV, Berkeley, Calif., 1968), pages 5-8. Amer. Math. Soc., Providence, R.I., 1970.

[2] A. Andronov and L. Pontrjagin. Systèmes grossieres. Dokl. Akad. Nauk. USSR, pages 247-251, 1937.

[3] D. V. Anosov. Geodesic flows on closed Riemann manifolds with negative curvature. Proceedings of the Steklov Institute of Mathematics, No. 90 (1967). Translated from the Russian by S. Feder. American Mathematical Society, Providence, R.I., 1969.

[4] D. V. Anosov. Structurally stable systems. Trudy Mat. Inst. Steklov., 169:59-93, 254, 1985.

[5] V. I. Arnold. Small denominators. Amer. Math. Soc. Transl., (2) 461950.

[6] H. F. DeBaggis. Dynamical systems with stable structures. Contributions to the Theory of Nonlinear Oscillations, vol. II, pp. 37-59. Princeton University Press, Princeton, 1952.

[7] G. D. Birkhoff. Collected mathematical papers (in three volumes). Vol. I: Boundary value problems and associated Sturmian problems, differential equations, difference equations, dynamics (partial). Editorial committee: D. V. Widder (Chairman), C. R. Adams, R. E. Langer, Marston Morse and M. H. Stone. Dover Publications, Inc., New York, 1968.

[8] C. Gutierrez. Structural stability for flows on the torus with a cross-cap. Trans. Amer. Math. Soc., 241:311-320, 1978. 
[9] C. Gutierrez. A counter-example to a $C^{2}$ closing lemma. Ergodic Theory Dynam. Systems, 7(4):509-530, 1987.

[10] D. Hilbert. Über das unendliche. Math. Ann., 95:161-190, 1926.

[11] M. W. Hirsch, C. C. Pugh, and M. Shub. Invariant manifolds. Lecture Notes in Mathematics, Vol. 583. Springer-Verlag, Berlin-New York, 1977.

[12] H. Kneser. Reguläre kurvenscharen auf den ringflächen. Math. Ann., 91:135-154, 1924.

[13] S. Lefschetz. Geometric differential equations: Recent past and proximate future. In Differential Equations and Dynamical Systems (Proc. Internat. Sympos., Mayaguez, P. R., 1965), pages 1-14. Academic Press, New York, 1967.

[14] S. T. Liao. On the stability conjecture. Chinese Ann. Math., 1(1):9-30, 1980.

[15] A. Liapounoff. Problème Général de la Stabilité du Mouvement. Annals of Mathematics Studies, no. 17. Princeton University Press, Princeton, N. J.; Oxford University Press, London, 1947. [16] R. Mañé. An ergodic closing lemma, Ann. of Math., 16:503-540, 1982.

[17] R. Mañé. A proof of the $C^{1}$ stability conjecture. Inst. Hautes Études Sci. Publ. Math., (66):161-210, 1988 .

[18] N. G. Markley. The Poincaré-Bendixson theorem for the Klein bottle. Trans. Amer. Math. Soc., 135:159-165, 1969.

[19] S. E. Newhouse. Nondensity of axiom A(a) on $S^{2}$. In Global Analysis (Proc. Sympos. Pure Math., Vol. XIV, Berkeley, Calif., 1968), pages 191-202. Amer. Math. Soc., Providence, R.I., 1970.

[20] J. Palis. On the $C^{1} \Omega$-stability conjecture. Inst. Hautes Études Sci. Publ. Math., (66):211-215, 1988.

[21] J. Palis and S. Smale. Structural stability theorems. In Global Analysis (Proc. Sympos. Pure Math., Vol. XIV, Berkeley, Calif., 1968), pages 223-231. Amer. Math. Soc., Providence, R.I., 1970 .

[22] M. C. Peixoto and M. M. Peixoto. Structural stability in the plane with enlarged boundary conditions. An. Acad. Brasil. Ci., 31:135-160, 1959.

[23] M. M. Peixoto. On structural stability. Ann. of Math. (2), 69:199-222, 1959.

[24] M. M. Peixoto. Some examples on $n$-dimensional structural stability. Proc. Nat. Acad. Sci. U.S.A., 45:633-636, 1959.

[25] M. M. Peixoto, On the classification of flows on 2-manifolds, In Dynamical systems (Proc. Sympos., Univ. Bahia, Salvador, 1971), pages 389-419 Academic Press, New York, 1973.

[26] M. M. Peixoto. Structural stability on two-dimensional manifolds. Topology, 1:101-120, 1962.

[27] M. M. Peixoto. On an approximation theorem of Kupka and Smale. J. Differential Equations, $3: 214-227,1967$.

[28] M. M. Peixoto. On bifurcations of dynamical systems. Proceedings of the International Congress of Mathematicians (Vancouver, B. C., 1974), Vol. 2, Canad. Math. Congress, Montreal, Que., pages 315-319, 1975.

[29] M. M. Peixoto and C. C. Pugh. Structurally stable systems on open manifolds are never dense. Ann. of Math. (2), 87:423-430, 1968.

[30] V. A. Pliss. Nonlocal problems of the theory of oscillations. Translated from the Russian by Scripta Technica, Inc. Translation edited by Harry Herman. Academic Press, New York-London, 1966.

[31] H. Poincaré. Ouevres Complets, Équations différentielles, Vol. 1. Éditions Jacques Gabay, 1928.

[32] H. Poincaré. L'avenir des mathématiques. Scientia (Milano), 110(5-8):357-368, 1975. Abridged text of a paper read at the Fourth International Congress of Mathematicians, Rome, 1908, With introductions in Italian and English.

[33] C. C. Pugh. The closing lemma. Amer. J. Math., 89:956-1009, 1967.

[34] C. C. Pugh. An improved closing lemma and a general density theorem. Amer. J. Math., $89: 1010-1021,1967$.

[35] J. W. Robbin. A structural stability theorem. Ann. of Math. (2), 94:447-493, 1971. 
[36] R. C. Robinson. $C^{r}$ structural stability implies Kupka-Smale. In Dynamical systems (Proc. Sympos., Univ. Bahia, Salvador, 1971), pages 443-449. Academic Press, New York, 1973.

[37] S. Smale. Morse inequalities for a dynamical system. Bull. Amer. Math. Soc., 66:43-49, 1960. [38] S. Smale. Diffeomorphisms with many periodic points. In Differential and Combinatorial Topology (A Symposium in Honor of Marston Morse), pages 63-80. Princeton Univ. Press, Princeton, N.J., 1965.

[39] S. Smale. Structurally stable systems are not dense. Amer. J. Math., 88:491-496, 1966.

[40] S. Smale. Differentiable dynamical systems. Bull. Amer. Math. Soc., 73:747-817, 1967.

[41] S. Smale. Stability and genericity in dynamical systems. Seminaire Bourbaki, 12:177-185, 1969-1970.

[42] S. Smale. The mathematics of time. Springer-Verlag, New York-Berlin, 1980. Essays on dynamical systems, economic processes, and related topics.

[43] J. Sotomayor. Generic one-parameter families of vector fields on two-dimensional manifolds. Inst. Hautes Études Sci. Publ. Math., 43:5-46, 1974.

[44] R. Thom. Sur une partition en cellules associée à une fonction sur une variété. C. R. Acad. Sci. Paris, 228:973-975, 1949.

[45] R. F. Williams. The "DA" maps of Smale and structural stability. In Global Analysis (Proc. Sympos. Pure Math., Vol. XIV, Berkeley, Calif., 1968), pages 329-334. Amer. Math. Soc., Providence, R.I., 1970. 


\section{Comentários de encerramento}

Nesta seção formularemos alguns comentários sobre o discurso de aceitação proferido por M. Peixoto por ocasião do prêmio recebido da TWAS (The Word Academy of Sciences) ${ }^{11}$ em 14 de setembro de 1987 em Pequim, e também sobre os impactos da sua fecunda obra matemática, a partir dos marcos de referência apontados na Figura 1.

3.1. Influências e interações matemáticas de $M$. Peixoto citadas no seu discurso de aceitação (D.A.) do prêmio da TWAS.

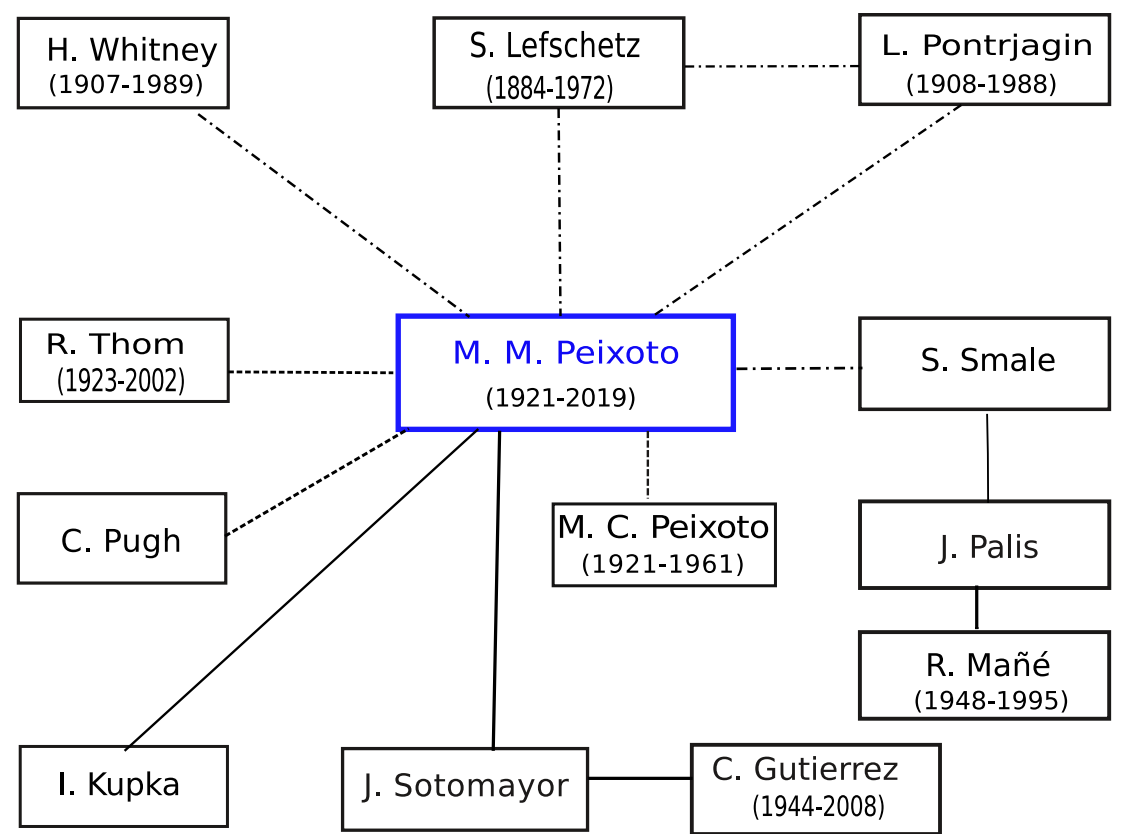

FigurA 3. Diagrama ilustrativo das influências e interações matemáticas de M. M. Peixoto citadas na Seção 2.

\subsection{Anotações dos tradutores sobre o D.A.}

3.2.1. Sobre o trabalho de DeBaggis. A prova usa o Teorema de Dulac: "Um campo vetorial polinomial no plano possui somente um número finito de órbitas fechadas isoladas (ciclos limites)."

Dulac é desnecessário. As provas deste teorema na literatura tem sido assimiladas por poucos leitores ${ }^{12}$. A construção do homeomorfismo da equivalência topológica em [6] é defeituosa. O trabalho [22] corrige este ponto.

\footnotetext{
${ }^{11}$ twas.org/recipients-twas-awards-and-prizes

12 images.math.cnrs.fr/L-histoire-mouvementee-des-cycles
} 
3.2.2. Sobre os trabalhos de 1959 ([22] e [23]). Uma prova da genericidade da estabilidade estrutural, que se aplica também para sistemas polinomiais, foi dada por [25, cap. 3], com versão abreviada em [26]. Essa prova não apela aos resultados de Dulac e nem de Petrovskii - Landis, cujas correções foram, em ambos os casos, refutadas.

3.2.3. Sobre as bifurcações. Os variados padrões de como as componentes conexas de $\Sigma$ incidem nos estratos de $\chi_{1}=\chi \backslash \Sigma$ está longe de ser entendida, mesmo para o caso local dos equilíbrios de codimensão quatro, cujos diagramas de bifurcações genéricas estão longe de serem cabalmente conhecidos. Para o já complexo caso de codimensão 3 , sugerimos [2].

\subsection{Sobre os marcos de referência da Figura 1 na Seção 1 .}

\subsubsection{Problemas de Equações Diferenciais, IMPA, 1962-64.}

Entre 1962 e 1964, M. Peixoto organizou um seminário sobre a Teoria Qualitativa das Equações Diferenciais. Entre os assuntos especiais desta atividade esteve a apresentação de trabalhos relacionados com seu artigo [26] Seção 2, sobre a Estabilidade Estrutural Bidimensional, sua possível extensão a dimensão superior e o estudo da vulneração das condições de estabilidade: a Teoria de Bifurcações. Jorge Sotomayor (J.S.) teve o privilégio de participar desse seminário deixando um registro dessa experiência única nos relatos evocativos [21] e [20]. O primeiro tem como ponto de partida o contato epistolar, em 1961, de um jovem que desejava inciar-se no estudo das equações diferenciais. O segundo se desenvolve em torno dos problemas de pesquisa em equações diferenciais propostos por M. Peixoto para os aspirantes a ingressar na área.

3.3.2. Simpósio Internacional de Sistemas Dinâmicos, 1971. Este extraordinário evento realizado em Salvador foi testemunhado por J.S. As atas desse evento foram editadas por Peixoto [16]. Trata-se de um notável marco de referência na área. Ver também [25], Seção 2.

3.3.3. Peixoto 80, IME - USP, 2001. Homenagem a Maurício Peixoto por ocasião do seu octagésimo aniversário. Sessão Especial da Academia Brasileira de Ciências. As atas deste evento foram editadas por J.S. Nas páginas 3-9 deste volume, publicado em [23], encontra-se o ensaio biográfico A few words about M. M. Peixoto, também depositado em www.ime.usp.br/ ${ }^{\sim}$ sotp/peixoto.pdf. Relacionadas diretamente com a obra de M. Peixoto, neste volume encontram-se contribuições de C. Gutierrez, R. Langevin et al., C. Pugh et al. e A. R. da Silva.

3.3.4. DYNA 2008. Universidade do Minho, Braga, Portugal - Dynamics, Games and Science. Evento em homenagem a Maurício Peixoto com um artigo de sua autoria [10]. Destacamos duas contribuições conectando o cerne da Seção 2 com assuntos cruciais apenas esboçados aí: o ensaio matemático e biográfico de C. Pugh sobre o Closing Lemma, veja [17], e o artigo de E. Pujals apontando para trabalhos atuais que, na Seção 2, são apenas sugeridos, entre os quais estão alguns de sua autoria e, também, aqueles pertinentes às Conjecturas de J. Palis. Ver [18]. 
3.3.5. Workshop Internacional de Sistemas Dinâmicos, IMPA, 2011. Citamos as seguintes palestras neste evento celebrando o nonagésimo aniversário de M. Peixoto: The work of Peixoto on Structural Stability as a paradigm for the Qualitative Theory of Differential Equations of Classical Geometry, proferida por J.S., disponibilizada em:

$$
\text { www.youtube.com/watch? } \mathrm{v}=\mathrm{JX} 2 \mathrm{pHiCvaxw} \text {. }
$$

Dynamical behavior of special curves on surfaces, proferida por Ronaldo Garcia (R.G.). Ver www.youtube.com/watch?v=7xT3hadM1-w.

3.3.6. Tributo a M. Peixoto, Unicamp, 2011. Palestra ${ }^{13}$ proferida por J.S. na celebração do nonagésimo aniversário de M. Peixoto, no evento Topological Methods on Dynamical Systems, 2011, UNICAMP. O texto da apresentação está depositado em www.researchgate.net/publication/325934473

Na parte final do vídeo estão gravados os depoimentos dos distintos matemáticos Djairo Guedes de Figueiredo, Alberto Verjovsky e Antônio R. da Silva que tiveram contato com M. Peixoto em fases diversas de suas carreiras.

3.3.7. Obras selecionadas de M. Peixoto, 2019. Publicação em preparação. Colaboração do IMPA e da SBM.

\subsection{Repercussões do trabalho de Peixoto: dois exemplos.}

3.4.1. Linhas de curvatura em superfícies. O trabalho pioneiro de M. Peixoto sobre a estabilidade estrutural de fluxos em superfícies encorajou estudos correlatos para as equações diferenciais da Geometria Clássica das superfícies, uma área cujas origens se remontam a Monge, Dupin, Darboux e Caratheodory, entre outros. No que diz respeito às linhas de curvatura e seus pontos singulares umbílicos de superfícies em $\mathbb{R}^{3}$, veja [5], [4] e [3]. Nesta última referência também são abordadas outras estruturas geométricas associadas a equações diferenciais, entre elas as linhas assintóticas em superfícies em $\mathbb{R}^{3}$ e as linhas de curvatura axial que são definidas para superfícies imersas em $\mathbb{R}^{4}$.

3.4.2. Regularização e estabilidade estrutural. O trabalho de M. Peixoto também influenciou o estudo das equações diferenciais descontínuas, ditas também de Filippov, via o procedimento de regularização, ou suavização. Citamos duas contribuições nesta direção: [22] e [24].

3.5. Sobre a Teoria de Decomposição Focal de M. Peixoto. M. Peixoto dedicou-se com afinco ao tema "decomposição focal" que tem como problema natural a análise do número de geodésicas ligando dois pontos numa variedade Riemanniana, ou mais geralmente, para uma equação diferencial de segunda ordem.

Listamos a seguir alguns trabalhos de M. Peixoto: [15], [14], [13], [12], [11] e [9]. Um comentário mais detalhado sobre essas contribuições será feito noutra ocasião.

${ }^{13}$ Vídeo disponível em www.dailymotion.com/video/xlqumf. 
3.6. Sistemas dinâmicos hiperbólicos. Em várias passagens da Seção 2, Peixoto aborda as contribuições de Smale sobre algumas extensões do seu Teorema [26], Seção 2. Para desenvolvimentos recentes nesta linha, sugerimos as seguintes fontes bibliográficas: [7], [6], [19] e [1].

3.7. Conclusão. Poder-se-ia perguntar como, a partir da modesta formação matemática recebida pelos engenheiros de sua época no Brasil, Maurício Peixoto desenvolveu a visão, penetração, sofisticação e o toque estético que é uma marca do seu trabalho matemático.

O fato é que ele pertenceu a uma geração de cientistas autodidatas - os líderesuma classe de espíritos talentosos especiais, vivendo à frente de seu tempo. Aqueles que por si mesmos definiram sua formação, abrindo seus próprios caminhos e apontando para novas direções.

Na entrevista publicada no livro, 14 lançado por ocasião do cinquentenário do IMPA, páginas 240-250, concedida em 2003, encontram-se interessantes dados biográficos e da carreira matemática de M. Peixoto, incluindo a fase anterior a 1955, ano referencial para o presente artigo. Ver figura 1.

A memória de Maurício Peixoto e o legado de sua obra são patrimônios vivos que palpitam em nossas mentes e corações. A descrição parcial aqui apresentada está longe de esgotar o vasto acervo que a respeito já foi publicado e de abranger o amplo panorama de suas contribuições.

\section{REFERÊNCIAS}

[1] C. Bonatti, Survey: Towards a global view of dynamical systems, for the $C^{1}$-topology. Ergodic Theory Dynam. Systems 31 (2011), no. 4, 959-993.

[2] F. Dumortier; R. Roussarie; J. Sotomayor and H. Zoladek, Bifurcations of planar vector fields. Nilpotent singularities and Abelian integrals. Lecture Notes in Mathematics, 1480. SpringerVerlag, Berlin, 1991. viii +226 pp.

[3] R. Garcia and J. Sotomayor, Differential Equations of Classical Geometry, a Qualitative Theory, Publicações Matemáticas, IMPA, $27^{0}$ Colóquio Bras. Mat., 2009, pp. $1-256$.

[4] C. Gutierrez and J. Sotomayor, An approximation theorem for immersions with stable configurations of lines of principal curvature, Geometric Dynamics (Proc. Rio de Janeiro, 1981), Lectures Notes in Math., vol. 1007, Springer-Verlag, 1983, pp. 332-368.

[5] C. Gutierrez and J. Sotomayor, Structurally stable configurations of lines of principal curvature, Astérisque 98-99 (1982), 195-215.

[6] J. Palis, Open questions leading to a global perspective in dynamics. Nonlinearity 21 (2008), no. 4, T37-T43.

[7] J. Palis, A global perspective for non-conservative dynamics. Ann. Inst. H. Poincaré Anal. Non Linéaire 22 (2005), no. 4, 485-507.

[8] J. Palis e W. de Melo, Introdução aos sistemas dinâmicos. Projeto Euclides. Instituto de Matemática Pura e Aplicada, Rio de Janeiro, 1978. viii +190 pp.

[9] C. A. A. de Carvalho, M. M. Peixoto, D. Pinheiro and A. A. Pinto, An asymptotic universal focal decomposition for non-isochronous potentials. Trans. Amer. Math. Soc. 366 (2014), no. 4, 2227-2263.

[10] M. M. Peixoto, A Brief Survey of Focal Decomposition, Dynamics, games and science. I, 1-10, Springer Proc. Math., 1, Springer, Heidelberg, 2011.

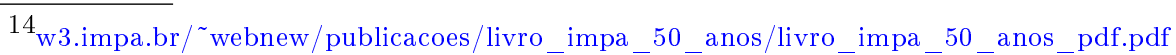


[11] M. M. Peixoto and C. Pugh, On focal stability in dimension two. An. Acad. Brasil. Ciênc. 79 (2007), no. 1, 1-11.

[12] I. Kupka, M. M. Peixoto and C. Pugh, Focal stability of Riemann metrics. J. Reine Angew. Math. 593 (2006), 31-72.

[13] M. M. Peixoto and A. R. da Silva, Focal decomposition and some results of S. Bernstein on the 2-point boundary value problem. J. London Math. Soc. (2) 60 (1999), no. 2, 517-547.

[14] M. M. Peixoto, Focal decomposition in geometry, arithmetic and physics. Geometry, topology and physics (Campinas, 1996), 213-231, de Gruyter, Berlin, 1997.

[15] M. M. Peixoto and R. Thom, Le point de vue énumératif dans les problèmes aux limites pour les équations différentielles ordinaires. II. C. R. Acad. Sci. Paris Sér. I Math. 303 (1986), no. 14, 693-698.

[16] M. M. Peixoto, Editor, Dynamical Systems, Proceedings of a Symposium held at Univ. of Bahia, Salvador, Br., 1971, Ac. Press, New York and London, 1973.

[17] C. Pugh, The Closing Lemma in Retrospect, Dynamics, games and science. I, $721-741$, Springer Proc. Math., 1, Springer, Heidelberg, 2011.

[18] E. R. Pujals, From Peixoto's theorem to Palis's conjecture, Dynamics, games and science. I, 743-746, Springer Proc. Math., 1, Springer, Heidelberg, 2011.

[19] E. R. Pujals, Some simple questions related to the $C^{r}$ stability conjecture. Nonlinearity 21 (2008), no. 11, T233-T237.

[20] J. Sotomayor, On a list of problems of ordinary differential equations, SPJMS, vol 13, no. 1, (2019), 177-194.

[21] J. Sotomayor, Mathematical Encounters, Notices ICCM, vol. 6, no. 2, (2018), 94 - 98.

[22] J. Sotomayor and A. L. F. Machado, Structurally stable discontinuous vector fields in the plane. Qual. Theory Dyn. Syst. 3 (2002), no. 1, 227-250.

[23] J. Sotomayor, Editor, The Geometry of Differential Equations and Dynamical Systems, Computational and Applied Mathematics, 20, 1-2, 2001.

[24] J. Sotomayor and M. A. Teixiera, Regularization of discontinuous vector fields. International Conference on Differential Equations (Lisboa, 1995), 207-223, World Sci. Publ., River Edge, NJ, 1998.

[25] J. Sotomayor, Curvas definidas por equações diferenciais no plano. $13^{\circ}$ Colóquio Brasileiro de Matemática. Instituto de Matemática Pura e Aplicada, Rio de Janeiro, 1981. iii +166 pp.

[26] J. Sotomayor, La mesure de l'ensemble de bifurcation des champs de vecteurs dans le plan. C. R. Acad. Sci. Paris Sér. I Math. 293 (1981), no. 8, 405-407.

Instituto de Matemática e Estatística

Universidade de São Paulo

Rua do MaTÃo, 1010

SÃo Paulo, CEP 05508-090, SP

Instituto de Matemática E Estatística

Universidade Federal de Goiás

Campus Samambaia

CEP 74690-900 GOIÂNIA - GO

Instituto de Matemática e Computação

Universidade FEDERAL de Itajubá

Avenida BPS 1303, Pinheirinho

CEP 37.500-903, ItajubÁ, MG.

Email address: sotp@ime.usp.br, ragarcia@ufg.br, lfmelo@unifei.edu.br 